\title{
Relato de caso
}

\section{Planejamento digital para cirurgia de aumento de coroa clínico estético - relato de caso}

\section{Digital planning for aesthetic clinical crown increase surgery - case report}

\author{
Iuri Dornelas Prates Freitas* \\ Isadora Rabello de Almeida** \\ Amanda Pinheiro Lopes ${ }^{* * *}$ \\ Luiz Paulo Carvalho Rocha*** \\ Simone Angélica de Faria Amormino ${ }^{* * * *}$
}

\section{Resumo}

A exposição excessiva de gengiva durante o sorriso é denominada como sorriso gengival. O fator etiológico dessa alteração é variado e sua identificação é fundamental para confecção de um plano de tratamento eficaz. O planejamento clínico pode ser realizado utilizando uma abordagem dinâmica e digital do sorriso. Sendo assim, software, exames imaginológicos, modelo virtual 3D e guia cirúrgico aumentam a qualidade e a precisão do tratamento, trazendo inúmeros benefícios para o paciente. Objetivo: demonstrar a importância do fluxo digital no planejamento e na correção do sorriso gengival. Relato de caso: paciente apresentou queixas em relação ao tamanho dos dentes e ao excesso de exposição de gengiva durante o sorriso. Após exames clínico e radiográfico, foi possível realizar o diagnóstico do fator etiológico como erupção passiva alterada (EPA). O tratamento de escolha foi o recontorno gengival associado à realização de osteotomia e osteoplastia. Para uma maior precisão do tratamento, foi confeccionado um guia cirúrgico duplo. Após o procedimento, a paciente foi orientada e medicada. Considerações finais: a abordagem digital do sorriso favoreceu um tratamento cirúrgico preciso, eficaz e satisfatório para a paciente, reestabelecendo assim a harmonia dento-gengivo-facial.

Palavras-chave: planejamento digital; cirurgia periodontal; erupção passiva alterada.

**** Doutorando. Departamento de Morfologia. Universidade Federal de Minas Gerais, Instituto de Ciências Biológicas. Belo Horizonte, Minas Gerais, Brasil.

Professora de Periodontia Clínica. Departamento de Odontologia. Faculdade Sete Lagoas, Sete Lagoas, Minas Gerais, Brasil. 


\section{Introdução}

A exposição excessiva de gengiva, ao sorrir, também conhecida como "sorriso gengival", é uma queixa comum nos consultórios odontológicos e uma demanda crescente dos pacientes que buscam uma melhoria estética ${ }^{1}$. A percepção antiestética do sorriso pode gerar danos no comportamento e na expressividade social do indivíduo e, com isso, reduzir sua confiança e sua autoestima ${ }^{2}$. Em suma, a estética do sorriso é composta pela relação harmônica e equilibrada entre dentes, gengiva e lábios. Além disso, durante o sorriso, a exposição de gengiva não pode ultrapassar $2 \mathrm{~mm}$, medida compreendida da borda inferior do lábio superior até a margem gengival dos dentes superiores ${ }^{2,3}$.

A etiologia do sorriso gengival é multifatorial e pode compreender hiperfunção dos músculos periorais, comprimento curto do lábio superior, problemas esqueléticos causados pelo excesso vertical de maxila e erupção passiva alterada (EPA $)^{4}$. Sendo assim, o tratamento do sorriso gengival é um desafio, por exigir uma abordagem multidisciplinar e um planejamento meticuloso ${ }^{5}$. De acordo com o fator etiológico, o tratamento pode abranger cirurgia ortognática, miectomia, toxina botulínica, ortodontia, reposicionamento labial, ácido hialorônico e cirurgia periodontal ${ }^{4,6}$.

A abordagem periodontal é realizada no caso de EPA, esta é caracterizada pela interrupção da migração apical correta da gengiva marginal, o que resulta no encobrimento de parte da coroa anatômica do dente. Na EPA, a margem gengival assume uma posição mais coronal na coroa anatômica e não se aproxima da junção cemento-esmalte $(\mathrm{JCE})^{7}$.

A análise da EPA é comumente feita em uma sondagem transmucosa, sob anestesia, por meio de uma sonda periodontal, em que se observam as relações entre os tecidos moles e duros. Esse procedimento deve ser realizado de maneira meticulosa, por um profissional experiente, para que não haja erro nas mensurações observadas. Atualmente, uma maneira segura e fidedigna de analisar os tecidos de inserção supracrestal é a tomografia computadorizada de feixe cônico (TCFC). Para a análise da EPA, a TCFC é uma ferramenta essencial, pois permite a análise periodontal de tecidos duros e moles, quando se usa um afastador ${ }^{8}$. Do mesmo modo, é feita a mensuração da distância da JCE até a crista alveolar, comprimento da coroa clínica e anatômica ${ }^{7}$.

A preservação e a integridade dos tecidos de inserção supracrestal são fundamentais para que haja aderência do epitélio juncional, da inserção conjuntiva a estrutura dentária e a manutenção da saúde periodontal ${ }^{9}$. Uma distância de $3 \mathrm{~mm}$ entre a crista óssea e a JCE, ou término de preparos protéticos, é idealmente desejada após um procedimento de aumento de coroa ( $2 \mathrm{~mm}$ de espaço biológico e $1 \mathrm{~mm}$ de profundidade do sulco gengival $)^{10}$. Sendo este espaço composto, em média, por 1,04 $\mathrm{mm}$ da inserção do tecido conjuntivo, $0,97 \mathrm{~mm}$ de epitélio juncional, resultando em $2 \mathrm{~mm}^{10}$.

A constante evolução tecnológica permitiu que a Odontologia experimentasse uma era digital, viabilizando a transição de um fluxo de trabalho tradicional para uma abordagem digital ${ }^{11}$. Dentre essas abordagens, destaca-se o Digital Smile Design (DSD), que possibilita o refinamento e a precisão dos planos de tratamento através da digitalização e edição do sorriso e das estruturas que o compõem ${ }^{12}$. Atualmente, esse protocolo permite que o profissional analise as proporções faciais, com a relação entre as informações intra e extrabucais. A partir dessa observação, é possível traçar eixos para estabelecer formato e contorno gengival adequados ${ }^{13}$. Depois da aquisição dessas informações, elas são organizadas em um software simples para apresentação de slides e o sorriso digital é criado através de desenhos sobre as fotos ${ }^{13}$. O DSD apresenta inúmeras vantagens, como redução do tempo clínico, precisão, previsibilidade, planejamento integrado, aproximação profissional-paciente, diagnóstico estético, redução de intercorrências pós-operatórias, aumentando a percepção diagnóstica e reduzindo erros ${ }^{13,14}$.

Outras ferramentas que podem integrar o fluxo de trabalho digital são os scanners intraorais. Esses dispositivos permitem o escaneamento dos arcos dentários, podendo ser aplicados em software para duplicação, manipulação e análise digital dos modelos, consequentemente, aumentando a precisão, a validade e a confiabilidade das medições ${ }^{15}$. A utilização da documentação dinâmica do sorriso, associada ao protocolo DSD, fornece diagnósticos mais eficientes, planos de tratamen- 
to mais consistentes e melhores resultados após a finalização do procedimento ${ }^{16}$.

O presente trabalho tem por objetivo apresentar um caso clínico de aumento de coroa clínico por razões estéticas, utilizando o fluxo de trabalho digital com o uso de guia cirúrgico periodontal.

\section{Relato de caso clínico}

Paciente do gênero feminino, com 24 anos de idade, natural de Belo Horizonte, procurou atendimento odontológico relatando queixas de insatisfação estética, coroas dos dentes anteriores superiores muito curtas, em especial os incisivos laterais, e excesso de exposição gengival.
Durante a anamnese, não houve relato de alterações sistêmicas, uso crônico de medicamento ou histórico de tabagismo. No exame clínico extraoral, não foram verificadas variações significativas, sendo realizadas fotografias para análise da face e exame do sorriso (Figura 1). Após as avaliações da paciente, o diagnóstico do sorriso gengival foi de EPA. Foi, então, planejado o aumento de coroa clínica nos dentes superiores, do 15 ao 25 . Foram avaliados os parâmetros periodontais de profundidade de sondagem, nível clínico de inserção, sangramento em sondagem e mobilidade. Os dados obtidos foram compatíveis com o diagnóstico de saúde periodontal com periodonto intacto.

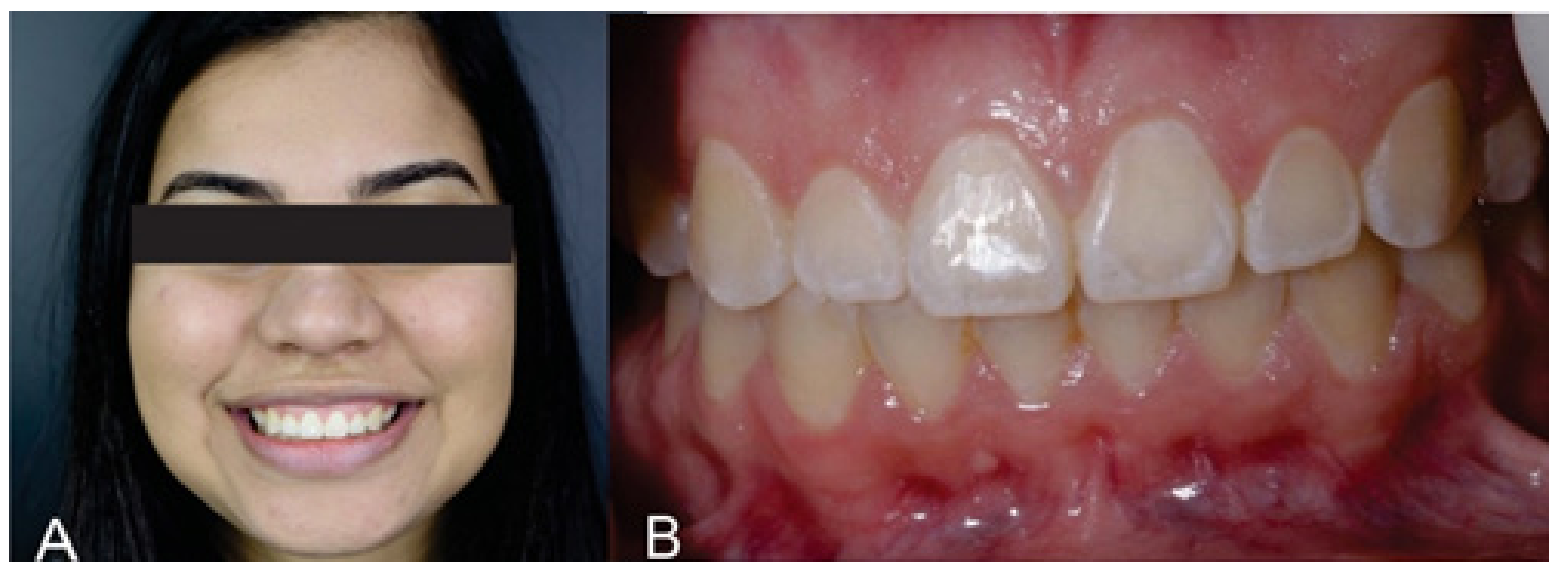

Figura 1 - A) Aspecto inicial da paciente (face) e B) aspecto inicial do sorriso (intraoral)

Fonte: autores.

O fluxo digital de trabalho foi realizado a fim de aumentar a previsibilidade da cirurgia e trazer segurança à paciente. Este fluxo consistiu-se de escaneamento intraoral e obtenção de uma TCFC (Figura 2), para um posterior planejamento digital do sorriso através de um software específico (3shape, Smile Desing Simulation) (Figuras 3 e 4). A partir disso, seguiu-se para a confecção de um guia cirúrgico duplo, feito em CadCam com base nas referências extraorais (linha mediana da face, linha bipupilar a comissura labial e linha da comissura lateral do olho à linha distal do canino) e intraorais (margem gengival à crista óssea e JCE e linha do sorriso) (Figura 5). O guia cirúrgico foi confeccionado em resina específica para impressões 3D (Guia Resina Dental SG). O planejamento foi aprovado e autorizado pela paciente.

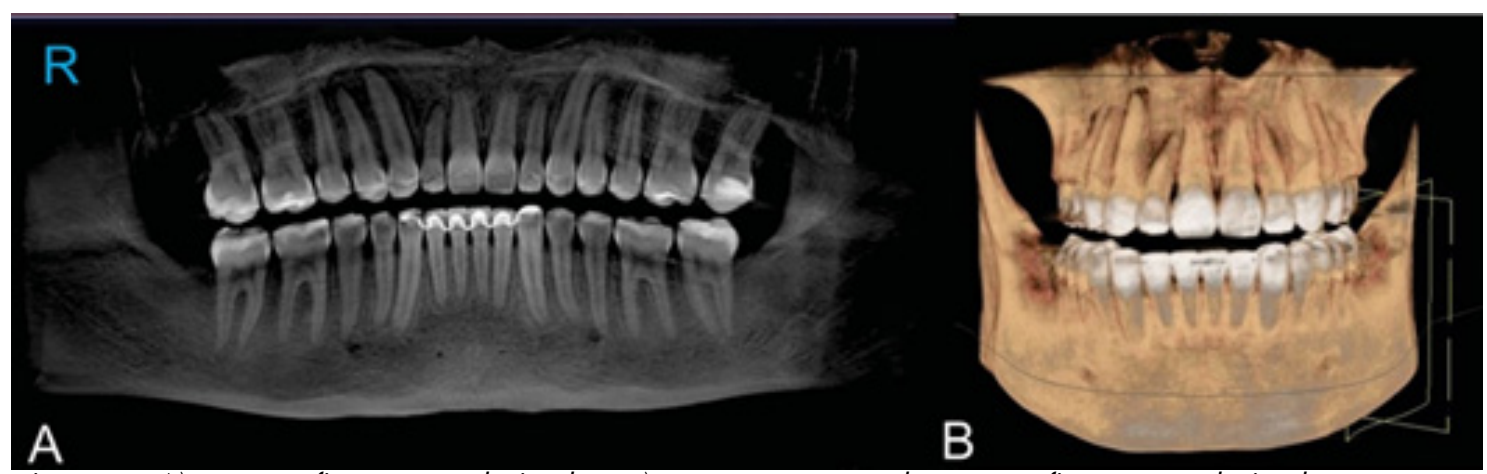

Figura 2 - A) Tomografia computadorizada e B) reconstrução 3D da tomografia computadorizada

Fonte: autores. 


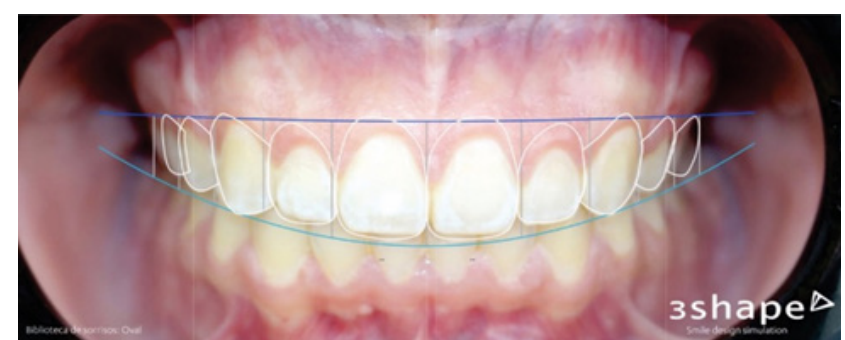

Figura 3 - Planejamento digital do sorriso (intraoral) Fonte: autores.

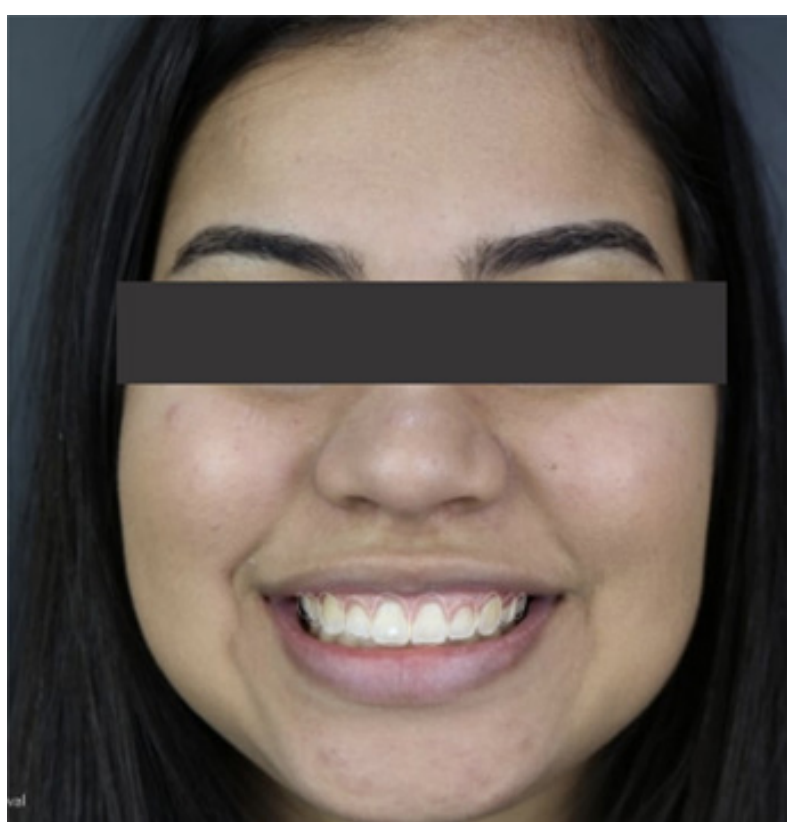

Figura 4 - Planejamento digital do sorriso (extraoral) Fonte: autores.

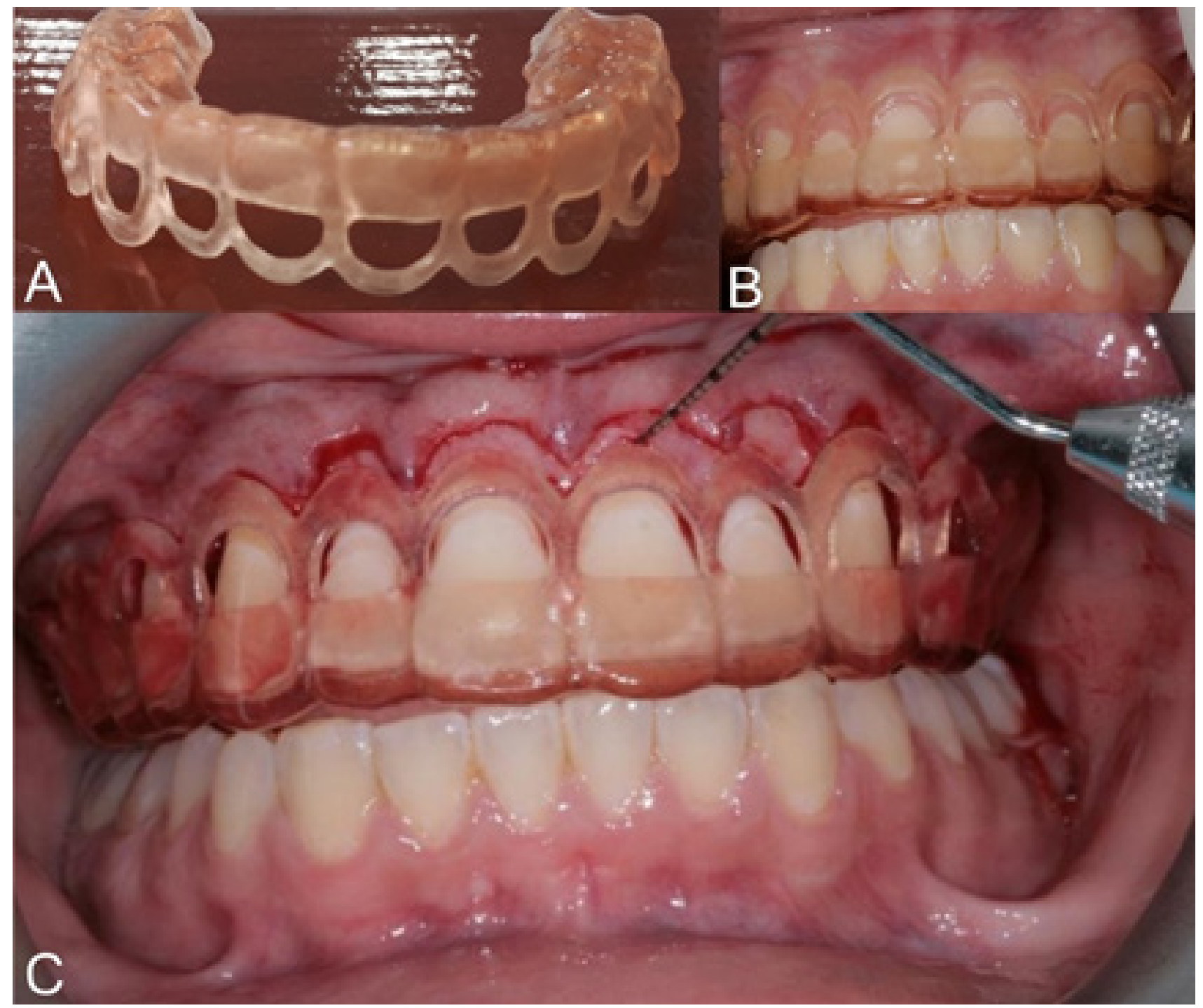

Figura 5 - A) Guia cirúrgico, B) guia cirúrgico instalado e C) demarcação do zênite Fonte: autores. 
Foi realizada assepsia extraoral com digluconato de clorexidina a $2 \%$, bochecho com clorexidina a $0,12 \%$, por um minuto, e anestesia infiltrativa com lidocaína a $2 \%$ com epinefrina 1:100.000. Com o guia cirúrgico em boca (Figura 5), foi realizado o bisel externo com lâmina $15 \mathrm{C}$ nos dentes 15 ao 25, nos espaços previamente determinados pelo guia. Posteriormente, retalho de espessura total, devido aos dados obtidos pela TCFC, no qual se verificou uma distância da gengiva aos espaços supracrestais menor que $1 \mathrm{~mm}$. Dessa forma, a osteotomia foi feita removendo $2 \mathrm{~mm}$ de cada dente com cinzel de Ochesenbein $\mathrm{n}^{\circ} 3$ e 4 e broca esférica 1014, acompanhando a anatomia de cada dente, e, com auxílio do guia, foi feita a demarcação do zênite (Figura 5).

Após o término da cirurgia, foram feitas as instruções de higiene oral, cuidados pós-operatórios e prescrição de fármacos para dor e bochecho com clorexidina $0,12 \%$ por 15 dias. A paciente foi acompanhada a cada 15 dias e reavaliada após 45 dias (Figura 6).

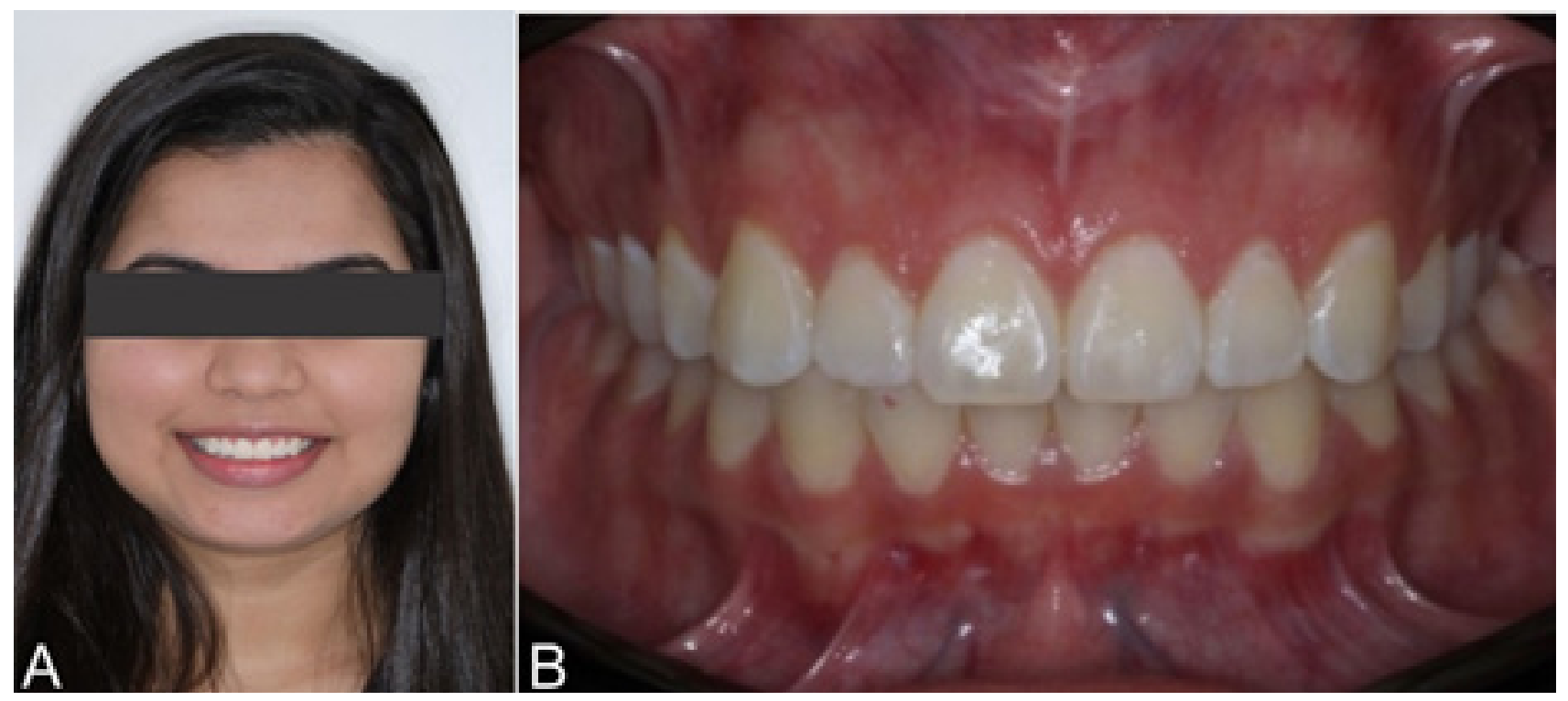

Figura 6 - A) Aspecto cirúrgico após 45 dias (sorriso espontâneo); B) aspecto cirúrgico após 45 dias (intraoral).

Fonte: autores.

\section{Discussão}

O sorriso gengival é caracterizado pela exposição excessiva de gengiva e pode comprometer a estética do sorriso ${ }^{17}$. Uma das causas do sorriso gengival é a EPA, que resulta na aparência de coroas clínicas curtas. Isso devido à variação morfológica do periodonto localizado mais incisal ${ }^{17}$.

O fator genético ainda é alvo de estudos, que tentam correlacionar o sorriso gengival como uma característica familiar. Em um estudo clínico preliminar, foi mostrada a associação da frequência do sorriso gengival no grupo familiar. Foi observado que $65 \%$ dos pacientes tinham pelo menos um ou mais membros da família com a mesma condição clínica ${ }^{18}$. Lembrando que a prevalência do sorriso gengival foi estimada em $10 \%$ dos in- divíduos de 20 a 30 anos, sendo mais comum em mulheres (14\%) do que em homens $(7 \%)^{19}$.

No presente caso, a EPA foi diagnosticada como fator principal do sorriso gengival da paciente. Diante disso, uma sequência de exames e análises se seguiu para o planejamento da cirurgia de aumento de coroa clínica. Então, com a TCFC, foi mensurada a relação da crista óssea à JCE, da margem gengival à crista óssea e JCE, além da altura da coroa clínica e anatômica ${ }^{7}$. Com bases nas informações obtidas, a paciente foi diagnosticada com EPA do tipo I e subtipo B, de acordo com o estudo realizado por Coslet et al. ${ }^{20}$.

A EPA foi morfologicamente dividida em dois tipos, de acordo com a localização da junção mucogengival à crista óssea, e os dois subtipos conforme a posição da crista óssea em relação à JCE. $\mathrm{Na}$ classificação, o tipo 1 se caracteriza pela ex- 
posição excessiva da margem gengival na coroa, e a junção mucogengival está situada mais apicalmente à crista óssea. Já o subtipo $\mathrm{B}$ é caracterizado pela íntima proximidade da crista óssea com a JCE ${ }^{17,19}$. Sendo assim, o tratamento da EPA é determinado de acordo com a distância da crista óssea à JCE ${ }^{17}$. No presente caso, a conduta clínica de escolha foi o recontorno gengival combinado com osteotomia e osteoplasia. Devido ao excesso de margem gengival, foi necessário realizar o recontorno gengival e a osteotomia, com a finalidade de devolver o espaço biológico, além disso, o perfil ósseo foi adequado com a osteoplasia.

$\mathrm{O}$ fenótipo gengival também tem grande influência no processo de recuperação dos tecidos gengivais supracrestais. Em um ensaio clínico com acompanhamento de doze meses, foi analisada a recuperação dos tecidos gengivais supracrestais no sentido coronal, mostrando que a reestruturação foi mais acentuada em paciente com fenótipo gengival espesso ${ }^{22}$. Em contrapartida, $75 \%$ dos pacientes com EPA apresentavam o fenótipo gengival espesso ${ }^{23}$. Logo, o paciente que apresenta o fenótipo gengival fino tem maiores possibilidades de recessão gengival, deiscência e fenestração ósseas, além de menor previsibilidade pós-cirúrgica em comparação ao fenótipo espesso $^{24}$. De acordo com o estudo de Rouck et al. ${ }^{25}$ (2009), a paciente apresentou o biótipo gengival do tipo A2 espesso e festonado. O biótipo gengival, normalmente, não é contraindicação de tratamento, no entanto, a condição sistêmica do paciente, tabagismo e uso de medicamentos podem ser empecilhos. Portanto, a anamnese bem-feita é essencial para o planejamento clínico. A EPA pode ser um fator de risco para a saúde, porque a profundidade do sulco aumentado contribui para aumento da retenção de biofilme dental, predispondo a outras doenças ${ }^{9}$.

O planejamento pelo DSD reduz as intercorrências durante o procedimento e no pós-operatório, não havendo limitações, exceto pela questão financeira do indivíduo. A paciente do presente caso poderia dispor de outras opções de tratamento, como a cirurgia de reposicionamento labial ou aplicação de toxina botulínica para redução da hiperfunção dos músculos periorais ${ }^{5,6}$.
O caso apresentado teve planejamento e execução otimizados com a ajuda de DSD, TCFC, escaneamento $3 \mathrm{D}$ e guia cirúrgico periodontal duplo. Os eixos como linha contorno gengival, bipupilar, sagital mediana e comissura labial e relação proporcional dente, lábio e gengiva foram considerados no DSD. Dessa forma, foi possível conduzir o procedimento com mais segurança e previsibilidade com base na literatura ${ }^{13,16}$. Nos últimos anos, com a variedade de aplicações e vantagens, os dispositivos de scanners intraorais vêm se tornando altamente interessantes e, consequentemente, mudando a realidade de planejamentos, diagnósticos e tratamentos odontológicos ${ }^{11,13}$.

O planejamento reverso é uma estratégia usada para proporcionar uma conduta mais segura ao clínico. A partir do escaneamento intraoral, foi possível confeccionar o guia cirúrgico periodontal duplo. Sendo muito importante, para definir o plano e a arquitetura gengival, estabelecer referências para a osteoplastia e a posição do zênite, estimando resultados mais confiáveis e eficientes, além de garantir a remoção segura e padronizada de osso ${ }^{26}$. Dessa forma, o cirurgião-dentista deve ter conhecimento sobre anatomia dental, estética branca e vermelha, além de princípios de proporções harmônicas dento-faciais e sua aplicação, para que possa visualizar e plotar os eixos estabelecidos como guia para recontorno gengival ${ }^{13}$.

A abordagem digital do sorriso é uma poderosa ferramenta de marketing, pois permite que $o$ paciente visualize o aspecto final do tratamento e que o cirurgião-dentista tenha a segurança de um procedimento com margem mínima de erro e intercorrências.

\section{Conclusão}

O sucesso da cirurgia de aumento de coroa clínico estético, com diminuição do sorriso gengival, restabelecimento dos tecidos de inserção supracrestal e harmonia dos tecidos dento-gengivais, apresenta uma maior previsibilidade com o fluxo digital de trabalho com guia cirúrgico periodontal e diagnóstico correto, além de tornar o plano de tratamento mais seguro, ágil e atraente para o paciente. 


\section{Abstract}

Excessive gum exposure during a smile is called a gum smile. The etiologic factor of this alteration is varied, the identification is essential to create an effective treatment plan. Clinical planning can be performed using a dynamic and digital smile approach. Therefore, software, imaging tests, 3D virtual model and the surgical guide increase the quality and precision of the treatment thus bringing numerous benefits to the patient. Objective: this case report aims to demonstrate the importance of digital flow in smile planning and correction of a gum smile. Case report: the patient presented complaints regarding tooth size and excessive gum exposure during the smile. After the clinical and radiographic examination, the etiological factor was diagnosed as an altered passive eruption (APE). The treatment of choice was the gingival contour associated with osteotomy and osteoplasty. For the precision of the treatment, a double surgical guide was made. After the procedure the patient was guided and medicated. Conclusion: the digital smile approach favored an accurate, effective and satisfactory surgical treatment for the patient thus restoring the tooth-gingival-facial harmony.

Keywords: digital planning; periodontal surgery; altered passive eruption.

\section{Referências}

1. Dym H, Pierre R. Diagnosis and Treatment Approaches to a "Gummy Smile". Dent Clin North Am 2020; 64(2):341-9.

2. Diaspro A, Cavallini M, Piersini P, Sito G. Gummy Smile Treatment: Proposal for a Novel Corrective Technique and a Review of the Literature. Aesthet Surg J 2018; 38(12):1330-8.

3. Nasr MW, Jabbour SF, Sidaoui JA, Haber RN, Kechichian EG. Botulinum Toxin for the Treatment of Excessive Gingival Display: A Systematic Review. Aesthet Surg J 2016; 36(1):82-8.

4. Hsien-Li Peng P, Peng JH. Treating the Gummy Smile With Hyaluronic Acid Filler Injection. Dermatol Surg 2019; 45(3):478-80

5. Tawfik OK, El-Nahass HE, Shipman P, Looney SW, Cutler $\mathrm{CW}$, Brunner M. Lip repositioning for the treatment of excess gingival display: A systematic review. J Esthet Restor Dent 2018; 30(2):101-12.

6. Chagas TF, Almeida NV, Lisboa CO, Ferreira DMTP, Mattos CT, Mucha JN. Duration of effectiveness of Botulinum toxin type A in excessive gingival display: a systematic review and meta-analysis. Braz Oral Res 2018; 32:e30.

7. Batista EL Jr., Moreira CC, Batista FC, de Oliveira RR, Pereira KK. Altered passive eruption diagnosis and treatment: a cone beam computed tomography-based reappraisal of the condition. J Clin Periodontol 2012; 39(11):1089-96.
8. Schertel Cassiano L, Barriviera M, Suzuki S, Giacomelli Nascimento G, Lourenço Januario A, Hilgert LA, et al. Soft tissue cone beam computed tomography (ST-CBCT) or the planning of esthetic crown lengthening procedures. Int J Esthet Dent 2016; 11(4):482-93.

9. Ferreira Júnior CD, Reis MMGC, Barboza ESP. Recuperação do espaço biológico: uma discussão sobre as medidas utilizadas nas cirurgias de aumento da coroa clínica com osteotomia. RGO Rev Gaúch Odontol 2013; 61(0):519-22.

10. Nautiyal A, Gujjari S, Kumar V. Aesthetic Crown Lengthening Using Chu Aesthetic Gauges and Evaluation of Biologic Width Healing. J Clin Diagn Res: JCDR 2016; 10(1):ZC51-5.

11. Brown GB, Currier GF, Kadioglu O, Kierl JP. Accuracy of 3-dimensional printed dental models reconstructed from digital intraoral impressions. Am J Orthod Dentofac 2018; 154(5):733-9.

12. Coachman C, Ricci A, Calamita M, Yoshinaga LG. Desenho digital do sorriso: do plano de tratamento à realidade clínica. In: Paolucci B. Visagismo: a arte de personalizar o desenho do sorriso. São Paulo: Vm Cultural; 2011. p. 147-62.

13. Coachman C, Calamita M. Digital smile design: A tool for treatment planning and communication in esthetic dentistry. Quintessence Dent Technol 2012; 35:103-11.

14. Okida RC, Moura AP, Franco LM, Salomão FM, Rahal V, Machado LS, et al. A The use of DSD (Digital Smile Design) for theoptimizationofthe dental esthetics. Rev Odontol 2017; 38(3):9-14.

15. Scott JD, English JD, Cozad BE, Borders CL, Harris LM, Moon AL, et al. Comparison of automated grading of digital orthodontic models and hand grading of 3-dimensionally printed models. Am J Orthod Dentofacial Orthop 2019; 155(6):886-90.

16. Stanley M, Paz AG, Miguel I, Coachman C. Fully digital workflow, integrating dental scan, smile design and CADCAM: case report. BMC Oral Health 2018; 18(1):134.

17. Alpiste-Illueca F. Altered passive eruption (APE): A little -known clinical situation. Med Oral Patol Oral Cir Bucal 2011; 1;16(1):e100-4.

18. Rossi R, Brunelli G, Piras V, Pilloni A. Altered passive eruption and familial trait: a preliminary investigation. Int $\mathrm{J}$ Dent 2014; 2014:874092.

19. Mele M, Felice P, Sharma P, Mazzotti C, Bellone P, Zucchelli G. Esthetic treatment of altered passive eruption. Periodontol 2000 2018; 77(1):65-83.

20. Coslet GJ, Vanarsdall R, Weisgold A. Diagnosis and classification of delayed passive eruption of the dentogingivaljunction in the adult. Alpha Omegan 1977; 10:24-8.

21. Al-Harbi F, Ahmad I. A guide to minimally invasive crown lengthening and tooth preparation for rehabilitating pink and white aesthetics. BDJ 2018; 224(4):228-34.

22. Pontoriero R, Carnevale G. Surgical crown lengthening: a 12-month clinical wound healing study. J Periodontol 2001; 72(7):841 $\square 8$.

23. Nart J, Carrió N, Valles C, Solís-Moreno C, Nart M, Reñé R, et al. Prevalence of Altered Passive Eruption in Orthodontically Treated and Untreated Patients. J Periodontol 2014; 85(11):e348-e53.

24. Zweers J, Thomas RZ, Slot DE, Weisgold AS, Van der Weijden FG. Characteristicsof periodontal biotype, its dimensions, associationsandprevalence: a systematicreview. J Clin Periodontol 2014; 41(10):958-71.

25. De Rouck T, Eghbali R, Collys K, De Bruyn H, Cosyn J. The gingival biotype revisited: transparency of the periodontal probe through the gingival margin as a method to dis- 
criminate thin from thick gingiva. J Clin Periodontol 2009; 36(5):428-33

26. Faria GJ, Barra SG, Vieira TR, de Oliveira PAD. A importância do planejamento multidiscipilinar para correção do sorriso gengival: relato de caso clínico. Faculdade Odontol Lins/Unimep 2015; 25(1):61-5.

Endereço para correspondência:

Simone Angélica de Faria Amormino

Avenida Brasil, 1.491, Sala 406 - Funcionários

CEP: 30140-002 - Belo Horizonte, MG, Brasil

E-mail: simoneamormino@hotmail.com

Recebido: 25/09/2020. Aceito: 05/04/2021. 